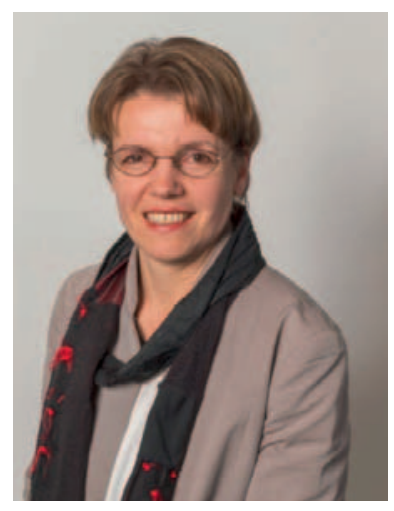

Dr. sc. nat.

Beatrix Falch,

Zürich, Schweiz

\title{
Kinder und Phytotherapie - ein heikles Thema?
}

Kinder sind in der Medizin eine besondere Patientengruppe, da für die meisten Medikamente keine klinischen Studien an Kindern (vor allem bis zum zwölften Lebensjahr) vorliegen. Dies soll sich zwar gemäss der EU-Verordnung 1901/2006 ändern, betrifft aber nur Neuzulassungen. Hinzu kommt, dass viele Eltern einen grossen Respekt gegenüber chemisch-synthetischen Medikamenten zeigen und die Rekrutierung von Kindern für klinische Studien oft auch an der fehlenden Einwilligung der Eltern scheitert. Somit bewegt man sich in der Praxis häufig im «Off-LabelUse», und der Anwendungserfahrung kommt eine grosse Bedeutung zu.

Zwar fehlen auch in der Phytotherapie in den meisten Fällen klinische Studien an Kindern gemäss «Good Clinical Practice» (GCP), aber im Vergleich zu chemisch-synthetischen Arzneimitteln kann in der Phytotherapie auf einen jahrhundertalten Erfahrungsschatz zurückgegriffen werden. Und letztendlich ist historisch betrachtet die heutige Schulmedizin aus der Phytotherapie hervorgegangen. Auch führen in der Schweiz noch zahlreiche (ca. 180) bei Swissmedic zugelassene pflanzliche Arzneimittel eine Kinderdosierung in der Patienteninformation und - falls vorhanden - auch in der Fachinformation auf. Eine aktualisierte Liste dieser Kinderdosierungen findet sich auf der Internetseite der Schweizerischen Medizinischen Gesellschaft für Phytotherapie (SMGP; www.smgp.ch).

Da Kinder im Vergleich zu Erwachsenen über hohe Selbstheilungskräfte und eine grosse Regenerationsfähig-

\section{KARGER}

Fax +497614520714

Information@Karger.com

www.karger.com
(C) 2015 S. Karger GmbH, Freiburg

$1015-0684 / 15 / 0275-0256 \$ 39.50 / 0$ keit verfügen, klingen viele Krankheiten viel schneller ab als bei Erwachsenen, auch wenn sie zuerst einen deutlich fulminanteren Verlauf zeigen. Daher reichen bei Kindern häufig mild wirksame Arzneimittel aus. Zudem bevorzugen auch Eltern die pflanzlichen bzw. «natürlichen» Arzneimittel, da sie deren Risiken als deutlich geringer einschätzen als jene von chemisch-synthetischen Arzneimitteln.

Somit ist die Phytotherapie eigentlich prädestiniert für die Therapie und die Beratung in der Pädiatrie. Denn einerseits liegt der Fokus in der Phytotherapie (und generell in der Komplementärmedizin) auf einer individuell zugeschnittenen Therapie, und andererseits reicht die eher milde Wirkung der pflanzlichen Arzneimittel für die Therapie von Erkrankungen bei Kindern meist vollkommen aus. Dennoch führt die Phytotherapie in der Kinderheilkunde ein Schattendasein - trotz hohem Sicherheitspotenzial und grosser therapeutischer Breite der meisten pflanzlichen $\mathrm{Zu}$ bereitungen. Häufig besteht nämlich unter Pädiatern eine gewisse Zurückhaltung der Phytotherapie gegenüber, da in neueren Arzneipflanzen-Monographien die Angaben zu Kinderdosierungen mangels entsprechender klinischer Daten fehlen, sodass eine naturwissenschaftliche Absicherung über diese Quelle nur schwer möglich ist. Ausserdem werden von schulmedizinischer Seite generell allergische oder sonstige unerwünschte oder unerwartete Reaktionen befürchtet, ohne dass diese belegt sind. Diese undifferenzierte Vorsicht gegenüber der Phytotherapie bis hin zur Ablehnung beruht häufig darauf, dass im Medizinstudium die Fächer Phytotherapie und Komplementärmedizin nicht gelehrt und nur an vereinzelten Hochschulen als Wahlfächer angeboten werden. Es besteht nun aber Hoffnung, dass sich dies in naher Zukunft ändern wird. Zurzeit wird nämlich der Lernzielkatalog für das Medizinstudium in der Schweiz überarbeitet, unter anderem mit dem Ziel, dass Studierende der Medizin neu auch eine gewisse Anzahl von Lektionen in Phytotherapie und Komplementärmedizin als Pflichtveranstaltungen besuchen müssen.

Weiterhin wird jedoch die Weiterbildung in Phytotherapie, wie sie die SMGP seit Jahren anbietet, notwendig sein. Denn erst mit dem Fähigkeitsausweis Phytotherapie, mit dem die Humanmedizinerinnen und -mediziner die Phytotherapie-Weiterbildung der SMGP abschliessen, hat man das nötige Rüstzeug, um kompetent Phytotherapie in der ärztlichen Praxis betreiben zu können, sofern man sich dieses Wissen nicht anderweitig angeeignet hat.

Der oben erwähnten Unsicherheit und Zurückhaltung gegenüber der Anwendung von pflanzlichen Arzneimitteln 
in der Kinderheilkunde möchte die SMGP nun mit ihrer 30. Schweizerischen Jahrestagung für Phytotherapie am 12. November 2015 in Baden zum Thema «Phytotherapie in der Pädiatrie und für stillende Mütter» mit fundierten Fachvorträgen entgegentreten. Mit dieser Tagung möchte die SMGP aufzeigen, dass nicht nur die in der SMGP vertretenen Pädiaterinnen und Pädiater sowie Apothekerinnen und Apotheker (hiervon treten einige als Referenten an der Tagung auf) tagtäglich beste Erfahrungen mit der Anwendung von pflanzlichen Arzneimitteln bei Kindern machen, sondern auch über die Schweizer Grenzen hinaus im deutschsprachigen Raum reichhaltige Praxiserfahrungen mit pflanzlichen Arzneimitteln zur Anwendung bei Kindern vorliegen. Referentinnen und Referenten aus Deutschland und Österreich, die alle aus dem Hochschuloder Klinikbereich stammen, werden dies an der Tagung im November belegen. Das genaue Tagungsprogramm findet sich auf der Internetseite der SMGP sowie auf der zweiten Umschlagseite dieser Ausgabe der ScHWEIZERISCHEN ZEITSCHRIFT FÜr GANZHEITSMEDIZIN.

Die SMGP hofft, dass sie mit dem thematisch breiten Tagungsprogramm und der Möglichkeit des fachlichen
Austauschs in den Pausen während der Tagung das Vertrauen in die Phytotherapie bei Kindern stärken und Anregungen für die Anwendung pflanzlicher Arzneimittel geben kann.

Zur Intensivierung des fachlichen Austauschs in der Phytotherapie führt die SMGP übrigens bereits seit einigen Jahren diverse Erfahrungsaustauschzirkel für Ärztinnen und Ärzte sowie Apothekerinnen und Apotheker durch. Seit 2011 existiert auch ein Erfahrungsaustauschzirkel der SMGP für Pädiatrie, der viermal im Jahr abwechselnd in Aarau und Zürich am Abend stattfindet - ein unkomplizierter und praxisnaher Einstieg in die Phytotherapie für Pädiater und an Kinderheilkunde interessierte Ärztinnen und Ärzte. Auch hierzu finden sich detaillierte Informationen unter: $w w w . s m g p . c h$.

Nun hoffe ich, Sie, liebe Leserinnen und Leser, auf die 30. Schweizerische Jahrestagung für Phytotherapie am 12. November 2015 in Baden neugierig gemacht zu haben, und ich würde mich freuen, wenn es der SMGP gelingt, durch Ihr zahlreiches Erscheinen an der Tagung auch nach aussen zu zeigen, wie wichtig die Phytotherapie in der täglichen medizinischen Praxis ist. 